\title{
EL ESTUDIO DE LA INTELIGENCIA ESPIRITUAL II: UN INSTRUMENTO DE EVALUAGIÓN DE SU DESEMPEÑO COMPETENCIAL
}

\section{THE STUDY OF SPIRITUAL INTELLIGENCE II: AN INSTRUMENT FOR EVALUATING THEIR COMPETENCE PERFORMANCE}

\author{
ANTONIA MARTÍN-SÁNCHEZ ${ }^{1}$, MÓNICA RODRÍGUEZ-ZAFRA², \\ Y JUAN CARLOS CENICEROS-ESTÉVEZ ${ }^{1}$
}

Cómo referenciar este artículo/How to reference this article:

Martín-Sánchez, A., Rodríguez-Zafra, M. y Ceniceros-Estévez, J. C. (2020). El estudio de la Inteligencia Espiritual II: un instrumento de evaluación de su desempeño competencial [The Study of Spiritual Intelligence II: An Instrument for Evaluating their Competence Performance].Acción Psicológica, 17(2), 103-122. https://doi.org/10.5944/ap.17.2.29528

\section{Resumen}

Este artículo se enmarca en la teoría de las Inteligencias Múltiples de Gardner (1983) que en 2012, demuestra que esta inteligencia cumple todos los criterios que determinan su incorporación al elenco de inteligencias. El estudio cualitativo de la Inteligencia Espiritual (IES) permitió determinar sus competencias: consciencia, transcendencia, amor, perdón, libertad, dolor-sufrimiento, sentido, gratitud y efectos. Tras la identificación de las competencias básicas, se ha construido el Cuestionario de
Inteligencia Espiritual (CIES) de 68 ítems y se ha validado en población española con una muestra de 528 participantes. Los resultados ponen de manifiesto su fiabilidad así como su validez de contenido, de constructo y de criterio. Se ha comprobado la unidimensionalidad del CIES y su capacidad de predicción. Las conclusiones son importantes en el nivel de intervención porque permiten identificar competencias claves para evaluar y desarrollar la IES mediante programas aplicados en distintos ámbitos educativos, sociales o empresariales.

Correspondence address [Dirección para correspondencia]: Mónica Rodríguez-Zafra, Facultad de Psicología, Universidad Nacional de Educación a Distancia, España.

Email: mrodriguez@psi.uned.es

ORCID: Antonia Martín-Sánchez (https://orcid.org/0000-0003-1090-310X), Mónica Rodríguez-Zafra (https://orcid.org/0000-0003-2609-0609) y Juan Carlos Ceniceros-Estévez (https://orcid.org/0000-0001-9348-8997).

${ }^{1}$ ESCUNI: Centro Universitario de Magisterio, España.

${ }^{2}$ Universidad Nacional de Educación a Distancia, España.

Recibido: 20 de junio de 2020.

Aceptado: 17 de diciembre de 2020. 
Palabras clave: Inteligencia Espiritual; Competencia; Investigación Cuantitativa.

\begin{abstract}
This study is about the 1983 Howard Gardner's theory of multiple intelligences (Gardner, 1983), in 2012 incorporates Spiritual Intelligence (SI or SQ) and demonstrates that it meets all the criteria that define each intelligence. The qualitative study of Spiritual Intelligence (IES) made it possible to determine its competences: consciousness, transcendence, love, forgiveness, freedom, pain-suffering, meaning, gratitude and effects. After such identification a 68 questions Spiritual Intelligence Questionnaire (SIQ) was built and validated with Spanish population with a sample of 528 participants. Results show the reliability and content, construct and criteria validity. Unidimensionality of the questionnaire was tested as well as his prediction capacity. The conclusions are important at the intervention level because they allow the identification of key competencies to evaluate and develop SI through programs applied in different educational, social, and business environments.
\end{abstract}

Keywords: Spiritual Intelligence; Competency; Quantitative Research.

\section{Introducción}

En Martín-Sánchez et al. (2020) se analizó con detalle la literatura existente sobre la Inteligencia Espiritual (IES) hasta el momento y se analizaron las propuestas teóricas y definiciones de los estudiosos de este tema llegándose a formular una definición inclusiva. Asimismo, en el estudio se analizaron y se detallaron, a través de un estudio cualitativo, las competencias que subyacen a la IES, su relevancia relativa y las relaciones entre ellas.

Tras esta investigación se define la Inteligencia Espiritual como un sistema de capacidades necesarias para la comprensión profunda de cuestiones de significado y sentido que hacen posible la integración de todas las dimensiones de la persona, la resolución de problemas existen- ciales, la trascendencia y la transformación de la realidad cotidiana a través de niveles altos de consciencia y de coherencia ética. Esta queda operativizada en nueve competencias: Consciencia, Trascendencia, Sentido, Compasión-amor, Aceptación del dolor, Perdón, Gratitud, Libertad y Efectos.

En este segundo estudio se presenta el proceso y los resultados de la construcción de un cuestionario para la medida de la IES y se recogen los resultados de la fase de verificación y contraste de los resultados.

\section{Método}

Al inicio de esta investigación se plantearon los siguientes objetivos específicos:

1. Operativizar y construir un instrumento de medida de la IES.

2. Validar dicho cuestionario.

3. Examinar las relaciones entre la IES, la resiliencia y la autoestima.

Gracias al análisis del estudio del concepto de IES realizado a partir de la literatura existente como a la investigación cualitativa recogida en Martín-Sánchez et al. (2020), se elaboró la primera versión del cuestionario. La triangulación en el análisis de las aproximaciones a la IES permite delimitar, concretar y definir cada competencia para la construcción del cuestionario. Se redactaron 118 ítems que operativizan las nueve competencias que componen la definición de la IES.

La primera versión del cuestionario se sometió a los siguientes análisis y comprobaciones de la calidad teórica y técnica para asegurar la validez y fiabilidad de los datos, antes de su aplicación definitiva:

- Validez del diseño y contenido del cuestionario.

- Análisis de la fiabilidad y consistencia interna del cuestionario (Prueba piloto).

- Validación de constructo.

El proceso de validación del contenido se realizó al someter el cuestionario a la técnica del juicio de expertos y, en concreto, al método de agregados individuales (Cabero y Llorente, 2013). Para ello, se pidió individualmente a 
cada experto que diera una estimación directa de la probabilidad de éxito o de fracaso en los datos demográficos propuestos y en cada uno de los ítems descriptores de las competencias de la IES. Con este fin, se elaboró el instrumento de evaluación del CIES para la validación de los expertos. En él, se les presentaba el objetivo de la investigación junto con los destinatarios de la misma, las secciones del instrumento (datos demográficos y los ítems asociados a cada una de las competencias estimadas de la IES) y las normas adicionales para la validación. A continuación, se les pidió que valorasen los ítems definitorios de cada competencia (la relevancia y claridad de cada uno de ellos con una escala del 1 al 5) e hicieran observaciones. Este documento fue enviado a seis expertos especialistas en Espiritualidad, Psicología y Métodos de Investigación. Todos los ítems valorados por debajo de cuatro puntos quedaron descartados y se recogieron todas las aportaciones realizadas por los expertos en la redacción de alguna de las afirmaciones. Por tanto, CIES quedó reducido a 104 ítems de 118 iniciales.

Después de la validación del diseño y de los contenidos del cuestionario por los seis expertos especialistas en Espiritualidad, Psicología y Métodos de Investigación, se valoró la fiabilidad y la consistencia interna del mismo a través de una prueba piloto. La muestra de esta prueba piloto estuvo formada por 53 participantes seleccionados por el procedimiento de bola de nieve. Para ello, se construyó un cuestionario online y se envió a un grupo inicial de participantes a los que se les pidió que lo reenviaran, a su vez, a otras personas. Una vez revisados los datos sociodemográficos de la muestra piloto, se calculó la fiabilidad total del cuestionario y la fiabilidad de cada una de las competencias. Se estimó el alfa de Cronbach global, 0.888, un valor alto que indica una fiabilidad elevada del cuestionario que permite deducir que todos los ítems miden un mismo aspecto. Para hacer el análisis y la selección de los ítems más representativos de cada competencia, se halló la correlación de cada ítem con el total. El criterio utilizado para seleccionar los ítems fue eliminar aquellos que menos correlacionasen con el total y su ausencia mejorase la consistencia interna alcanzada. El alfa de Cronbach global fue, en esta prueba piloto, de 0.888 con los 104 ítems y de 0.941 con los 68 ítems finalmente seleccionados. El índice de fiabilidad de las competencias de esta última versión del CIES se sitúa en un intervalo que va del 0.652 a 0.921.
Una vez depurado el cuestionario con los datos obtenidos en la prueba piloto, se procedió a la última fase de validación del cuestionario. Se construyó la versión definitiva del CIES online y se envió a una serie de participantes y se les pidió que lo reenviasen, a su vez, a otras personas, siguiendo el procedimiento de muestreo no probabilístico, como en la prueba piloto. Se optó por un muestreo de bola de nieve y, por tanto, el criterio fundamental en la selección de la muestra fue la accesibilidad. En los envíos iniciales se buscó informantes heterogéneos, representativos de diversas corrientes espirituales, edades, sexos, niveles económicos y formativos. Se garantizó el anonimato de los participantes y la confidencialidad de los datos demográficos pedidos y el respeto a los principios éticos que toda investigación ha de respetar (Martínez-Arias et al., 2014).

Se utilizó el paquete estadístico SPSS Statistics 24 para el análisis de los datos recogidos en la base de datos. Para el análisis diferencial se tuvo en cuenta un nivel de confianza del $95 \%$.

\section{Muestra}

La muestra final fue de 528 participantes, 132 hombres y 396 mujeres, lo que representa en porcentajes el $25 \%$ y el $75 \%$ respectivamente. La variabilidad de las edades de los participantes osciló entre los 18 y los 88 años, situándose la media en 47 años de edad, con una desviación típica de 13. Respecto al nivel económico, el $76 \%$ no superaba un salario de 2000 euros mensuales y menos del 6\% superaba los 3000 euros mensuales. El nivel de estudio de los participantes era alto: el $81 \%$ tenía formación superior y/o postgrado; tan solo el 3\% tenía estudios primarios. Respecto a la corriente religiosa o religión, el grupo más numeroso se definió como cristiano católico con un $68 \%$, seguido de los que afirman que no se adscribían a ninguna religión con un $20 \%$. El $2 \%$ reconoció que practicaba todas o que se quedaba con "lo mejor de cada una". Las otras religiones completan el resto. La media de personas con las que se convivían los participantes era de dos; y, respecto al lugar de residencia, el $81 \%$ de la muestra residía en una ciudad frente al $19 \%$ que residía en un pueblo. 


\section{Instrumentos de evaluación}

Adicionalmente al Cuestionario CIES, antes descrito, se utilizaron dos cuestionarios para el estudio de las relaciones entre la IES, la autoestima y la resiliencia.

Cuestionario de Autoestima de Rosenberg -RSQ(Rosenberg, 1965). Este cuestionario tiene por objetivo evaluar el sentimiento de satisfacción que una persona tiene sobre sí misma. Consta de 10 ítems que puntúan de 1a 4 en una escala tipo Likert. La fiabilidad test-retest es de $r=.85$ y el coeficiente alpha de consistencia interna es muy alto $\alpha=0.92$; (Fernández-Montalvo y Echeburúa, 2008). La validez convergente y discriminante es satisfactoria.

Escala de Resiliencia CD-RISC-10. Es un instrumento desarrollado por Serrano-Parra et al. (2013) derivada de la escala de resiliencia CD-RISC de Connor y Davidson (2003). Consta de 10 ítems y una sola dimensión con un sistema de respuesta tipo Likert de 0 a 4 . La estructura factorial se comprobó mediante análisis factorial confirmatorio que mostraba aceptables valores de bondad de ajuste tanto en hombres como en mujeres. La consistencia interna de los ítems se realizó mediante el $\alpha$ de Cronbach que fue de 0.81 . Respecto a la validez convergente, las puntuaciones globales de la versión española del CDRISC-10 correlacionaron directamente con el MOS y los componentes físico y mental del SF-12, e inversamente con las de PSS y GDS. Por tanto, la versión española del CD-RISC-10 mostró buenas propiedades psicométricas.

\section{Procedimientos estadísticos}

Se realizó un análisis descriptivo de las puntuaciones de la IES y del resto de variables y un análisis inferencial para encontrar posibles diferencias entre las variables. Para la validez de constructo se aplicó el Análisis Factorial Exploratorio-EFA y, para verificar la validez de criterio, se calculó el coeficiente de correlación Rho de Spearman y se confirmaron las correlaciones en las puntuaciones de cada sujeto con las medidas de Autoestima y Resiliencia.

\section{Resultados}

Los datos de las características de la muestra en cuanto a nivel económico, adscripción a religiones y distribución urbano-rural, fue similar a la reportada por el Instituto Nacional de Estadística (INE), el Centro de Investigaciones Sociológicas (CIS) y por el Banco Mundial para la población española.

Respecto al análisis de la fiabilidad y consistencia interna del CIES, los datos avalan la consistencia interna del cuestionario. El alfa de Cronbach global fue de 0.925. La consistencia interna de las subescalas de capacidad también fue alta: Consciencia 0.680; Trascendencia 0.902; Sentido 0.748; Amor 0.678; Perdón 0.772; Gratitud 0.785; Aceptación del Dolor 0.741; Libertad 0.781 y Efectos 0.751 .

Estos resultados son similares a los encontrados por Amran y Dryer (2008) en la validación de su Escala ISIS. El alfa de Cronbach global fue de 0.97 y los valores alfa de Cronbach para las subescalas fueron de 0.62 a 0.88 , con un valor promedio de 0.75 . Asimismo, otra escala de la IES, la Escala de SISRI (King, 2008) presentó un alfa de Cronbach global de 0.83 y sus cuatro dimensiones del mismo oscilaron entre 0.81 y 0.89 .

Además, estos datos corroboran los obtenidos en el marco teórico y en el estudio cualitativo previo (Rodríguez-Zafra et al., 2020). Cada una de estas competencias fue reconocida prácticamente por los principales investigadores de la IES como una competencia de la misma (Alonso, 2011; Amram, 2007; Chaktoura 2015; Cloninger, 2004; Day 2006; Draper, 2010; Emmons, 2000; Gómez 2014; Jahani, 2010; King y DeCicco, 2009; MacGilchrist et al., 2004; Maxim, 2008; Nasel, 2004; Noble, 2000; Ronel, 2008; Torralba 2010; Vaughan, 2002; Vázquez, 2012; Villafranca, 2016; Wigglesworth, 2013a, 2013b; Wolman, 2001; Zohar y Marshall, 2000) y por los participantes en la investigación cualitativa, con un número significativo de citas en la codificación del Atlas.ti.7.

El Análisis Factorial Exploratorio (AFE) permitió comprobar la validez de constructo. Se determinó la fiabilidad (consistencia interna) con un alpha de Cronbach de 0.925. Para las distintas competencias, un alpha de 
Cronbach entre 0.650 y 0.750 es aceptable y entre 0.750 y 0.899 es muy bueno. Todas las dimensiones quedaron en este último intervalo o muy próximas después de la reorganización de los ítems.

Previo al Análisis Factorial Exploratorio (AFE), se comprobó la factibilidad del procedimiento en este caso, calculando el Determinante de R, la esfericidad de Bartlett y el KMO. El determinante obtenido fue de prácticamente 0 , el valor $\mathrm{KMO}$ fue muy próximo a 1 y la significación de la prueba de esfericidad de Bartlett fue menor de 0.001. Todos los datos indican que el AFE era apropiado para el estudio de la estructura subyacente al cuestionario.

Igualmente, se determinó la unidimensionalidad del cuestionario siguiendo los criterios de Lima et al. (2012):

- Todos los ítems tienen que tener una $r$ de Pearson mayor que 0.30 en el primer factor durante la extracción. El primer factor explica una proporción importante de varianza con respecto a los demás factores.

Tabla 1.

Resultados del análisis factorial del cuestionario definitivo
- La varianza total explicada por los factores principales extraídos debe ser mayor del $50 \%$.

En el procedimiento de factorización aparecen 14 factores con una varianza total explicada del $58.12 \%$. Se ha seguido el criterio de Hair et al. (2007). En la Tabla 1 se muestra el resultado.

El paso siguiente fue comprobar la agrupación de los 68 ítems en los 14 factores en la matriz obtenida a partir de la rotación Varimax. La dispersión de los datos permitió la agrupación de los ítems. Estos estaban bien representados, con valores superiores a 0.5 en la gran mayoría de los casos o próximos a este valor. Tras la distribución y agrupación de los factores por competencias, se obtuvo la última versión del cuestionario.

\begin{tabular}{|c|c|c|c|c|c|c|}
\hline \multirow{2}{*}{ Componentes } & \multicolumn{3}{|c|}{ Autovalores iniciales } & \multicolumn{3}{|c|}{$\begin{array}{l}\text { Sumas de las saturaciones al cuadrado de } \\
\text { la extracción }\end{array}$} \\
\hline & Total & $\begin{array}{l}\text { \% de la } \\
\text { varianza }\end{array}$ & $\%$ Acumulado & Total & $\begin{array}{c}\text { \% de la } \\
\text { varianza }\end{array}$ & \% acumulado \\
\hline 1 & 14.720 & 21.647 & 21.647 & 14.720 & 21.647 & 21.647 \\
\hline 2 & 4.746 & 6.979 & 28.626 & 4.746 & 6.979 & 28.626 \\
\hline 3 & 3.384 & 4.977 & 33.602 & 3.384 & 4.977 & 33.602 \\
\hline 4 & 2.368 & 3.483 & 37.085 & 2.368 & 3.483 & 37.085 \\
\hline 5 & 2.139 & 3.145 & 40.230 & 2.139 & 3.145 & 40.230 \\
\hline 6 & 1.830 & 2.692 & 42.922 & 1.830 & 2.692 & 42.922 \\
\hline 7 & 1.598 & 2.350 & 45.272 & 1.598 & 2.350 & 45.272 \\
\hline 8 & 1.513 & 2.226 & 47.498 & 1.513 & 2.226 & 47.498 \\
\hline 9 & 1.404 & 2.065 & 49.563 & 1.404 & 2.065 & 49.563 \\
\hline 10 & 1.309 & 1.924 & 51.487 & 1.309 & 1.924 & 51.487 \\
\hline 11 & 1.194 & 1.757 & 53.244 & 1.194 & 1.757 & 53.244 \\
\hline 12 & 1.151 & 1.693 & 54.936 & 1.151 & 1.693 & 54.936 \\
\hline 13 & 1.098 & 1.615 & 56.552 & 1.098 & 1.615 & 56.552 \\
\hline 14 & 1.068 & 1.571 & 58.123 & 1.068 & 1.571 & 58.123 \\
\hline 67 & .203 & .298 & 99.712 & & & \\
\hline 68 & .196 & .288 & 100.000 & & & \\
\hline
\end{tabular}


Tabla 2.

Resultados descriptivos generales del cuestionario, por competencias

\begin{tabular}{|c|c|c|c|c|c|c|c|}
\hline & Media & $\begin{array}{c}\text { Desviación } \\
\text { estándar }\end{array}$ & Mínimo & Máximo & $\begin{array}{c}\text { Percentiles } \\
25 \\
\end{array}$ & $\begin{array}{c}\text { Percentiles } \\
50 \\
\end{array}$ & $\begin{array}{c}\text { Percentiles } \\
75 \\
\end{array}$ \\
\hline Consciencia & 4.78 & 0.66 & 1.50 & 6.00 & 4.33 & 4.83 & 5.17 \\
\hline Trascendencia & 4.36 & 1.09 & 1.00 & 6.00 & 3.75 & 4.58 & 5.29 \\
\hline Sentido & 5.13 & 0.69 & 2.00 & 6.00 & 4.80 & 5.20 & 5.60 \\
\hline Compasión-amor & 4.64 & 0.63 & 2.11 & 6.00 & 4.22 & 4.67 & 5.11 \\
\hline Perdón & 2.96 & 0.88 & 0.33 & 5.83 & 2.33 & 2.83 & 3.50 \\
\hline Gratitud & 5.26 & 0.61 & 2.57 & 6.00 & 4.86 & 5.43 & 5.71 \\
\hline Dolor & 4.35 & 0.81 & 1.00 & 6.00 & 3.80 & 4.40 & 5.00 \\
\hline Libertad & 4.90 & 0.68 & 2.00 & 6.00 & 4.55 & 5.00 & 5.44 \\
\hline Efectos & 5.22 & 0.56 & 0.75 & 6.00 & 4.87 & 5.25 & 5.62 \\
\hline Global & 4.62 & 0.48 & 3.12 & 5.76 & 4.31 & 4.68 & 5.00 \\
\hline
\end{tabular}

\section{Análisis descriptivos sobre las competencias de la Inteligencia Espiritual}

Como se observa en la Tabla 2 la competencia más valorada fue la Gratitud, seguida de los Efectos o consecuencias derivados de una alta inteligencia espiritual. Además de estas dos, Sentido, Libertad, Consciencia y Compasiónamor se encuentran por encima de la media; Trascendencia, Aceptación del dolor y Perdón por debajo. Esta última es la que obtuvo menor puntuación, con una diferencia significativa superior a dos puntos. Como se señaló anteriormente, los ítems del factor perdón están formulados de forma negativa, mientras que todos $-\mathrm{o}$ casi todo el restode ítems están formulados de forma positiva.

\section{Análisis diferencial sobre el desempeño de la Inteligencia Espiritual}

El análisis diferencial de las categorías cualitativas y cuantitativas, permitió comparar los resultados con otras investigaciones y variables sociodemográficas (sexo, edad, nivel económico, nivel formativo, estructura familiar). En los resultados obtenidos al comparar el nivel de estudios con los ingresos económicos, se observó que los participantes con un nivel formativo postgrado recibían más ingresos que el resto. Al comparar el nivel de ingresos por sexo, se observó que los hombres tenían salarios más altos que las mujeres. Estos datos correlacionan con la Encuesta de Condiciones de Vida (ECV) de 2016, publicada por el INE que son consistentes con los obtenidos en la presente investigación.

Los resultados presentados en la Tabla 3 muestran las diferencias significativas encontradas en la relación entre las puntuaciones obtenidas en las diferentes competencias según las principales variables analizadas.

Las mujeres puntuaron más alto en las competencias: Transcendencia, Compasión-amor, Perdón y Gratitud. Los hombres, en cambio, obtuvieron valores más altos en Libertad y Dolor. A partir del nivel de formación media y hasta la formación de postgrado se observó una progresión creciente en Consciencia, Transcendencia, Libertad y Dolor.

La religión Baha'i-Islam destaca en Trascendencia, Sentido, Compasión-amor, Libertad, Dolor y en la puntación global. Este grupo estaba configurado, sobre todo, por miembros de la religión Baha'i y por la rama sufí del islamismo; Las religiones Orientales destacaron en Trascendencia, Libertad, Dolor y en la puntación global; los cristianos ortodoxos puntuaron más alto en Trascendencia, Sentido y en la puntuación global, mientras que los cristianos católicos puntuaron más alto en Trascendencia, Sentido y Compasión-amor. Las personas que se definieron como practicantes de todas las religiones (Todas) obtuvieron puntuaciones altas en Transcendencia, Dolor y en la puntuación global. 
Tabla 3.

Resumen de los resultados del análisis diferencial de categorías cualitativas y cuantitativas

\begin{tabular}{|c|c|c|c|c|c|}
\hline & Sexo & $\begin{array}{c}\text { Nivel } \\
\text { económico }\end{array}$ & $\begin{array}{c}\text { Nivel } \\
\text { educativo }\end{array}$ & Religión & Convivencia \\
\hline & \multicolumn{5}{|c|}{ Mayor puntuación en la competencia } \\
\hline Transcendencia & Mujeres & & $\begin{array}{l}\text { a mayor nivel } \\
\text { educativo }\end{array}$ & $\begin{array}{c}\text { Católicos, } \\
\text { Islam, Orientales, } \\
\text { Ortodoxos, Todas }\end{array}$ & $\begin{array}{c}\text { en piso compartido, en } \\
\text { comunidad }\end{array}$ \\
\hline Consciencia & & $\begin{array}{l}\text { a mayor nivel } \\
\text { económico }\end{array}$ & $\begin{array}{l}\text { a mayor nivel } \\
\text { educativo }\end{array}$ & & \\
\hline Sentido & & $\begin{array}{l}\text { a mayor nivel } \\
\text { económico }\end{array}$ & & $\begin{array}{c}\text { Católicos, } \\
\text { Islam, Orientales, } \\
\text { Todas }\end{array}$ & en piso compartido, solos \\
\hline $\begin{array}{l}\text { Compasión } \\
\text { lamor }\end{array}$ & Mujeres & & & $\begin{array}{l}\text { Católicos, } \\
\text { Ortodoxos, Islam }\end{array}$ & $\begin{array}{c}\text { en piso compartido, en } \\
\text { comunidad }\end{array}$ \\
\hline Dolor & & & $\begin{array}{l}\text { a mayor nivel } \\
\text { educativo }\end{array}$ & $\begin{array}{c}\text { Islam, } \\
\text { Orientales, Todas }\end{array}$ & $\begin{array}{l}\text { en piso compartido, en } \\
\text { comunidad }\end{array}$ \\
\hline $\begin{array}{l}\text { Perdón } \\
\text { Gratitud }\end{array}$ & $\begin{array}{l}\text { Mujeres } \\
\text { Mujeres }\end{array}$ & & & & \\
\hline Libertad & & $\begin{array}{l}\text { a mayor nivel } \\
\text { económico }\end{array}$ & $\begin{array}{l}\text { a mayor nivel } \\
\text { educativo }\end{array}$ & Orientales & en piso compartido, solos \\
\hline $\begin{array}{l}\text { Puntuación } \\
\text { Global }\end{array}$ & Mujeres & & $\begin{array}{l}\text { a mayor nivel } \\
\text { educativo }\end{array}$ & $\begin{array}{l}\text { Islam, Orientales, } \\
\text { Ortodoxos, Todas }\end{array}$ & $\begin{array}{c}\text { en piso compartido, en } \\
\text { comunidad }\end{array}$ \\
\hline
\end{tabular}

Las personas que compartían piso o vivían en comunidad puntuaron significativamente más alto en Trascendencia, Sentido, Compasión-amor, Dolor y en la puntuación global.

\section{Tabla 4.}

Análisis de correlaciones. Competencias

\begin{tabular}{|c|c|c|c|c|c|c|c|}
\hline & 1 & 2 & 3 & 4 & 5 & 6 & 7 \\
\hline 1. Transcendencia & - & & & & & & \\
\hline 2. Sentido & $.510^{* *}$ & - & & & & & \\
\hline 3. Compasión-Amor & $.568^{* *}$ & $.461^{* *}$ & - & & & & \\
\hline 4. Gratitud & $.499^{* *}$ & $.599^{* *}$ & $.545^{\star *}$ & - & & & \\
\hline 5. Dolor & $.535^{\star *}$ & $.578^{* *}$ & $.494^{\star *}$ & $.542^{* *}$ & - & & \\
\hline 6. Libertad & $.455^{* *}$ & $.639^{\star *}$ & $.425^{\star *}$ & $.598^{\star *}$ & $.670^{* *}$ & - & \\
\hline 7. Efectos & $.381^{* *}$ & $.608^{* *}$ & $.482^{* *}$ & $.645^{\star *}$ & $.568^{* *}$ & $.598^{* *}$ & - \\
\hline GLOBAL & $.824^{* *}$ & $.716^{\star *}$ & $.758^{* *}$ & $.739^{* *}$ & $.723^{* *}$ & $.724^{* *}$ & $679^{* *}$ \\
\hline
\end{tabular}

En cuanto a las relaciones entre las competencias la Tabla 4 muestra los resultados de las correlaciones de cada una de ellas con el resto. 
Tabla 5.

Matriz de distancias obtenidas entre las competencias de la IES.

\begin{tabular}{|c|c|c|c|c|c|c|c|c|c|c|}
\hline \multirow{2}{*}{\multicolumn{2}{|c|}{ Caso }} & \multicolumn{9}{|c|}{ Archivo matricial de entrada } \\
\hline & & 1 & 2 & 3 & 4 & 5 & 6 & 7 & 8 & 9 \\
\hline 1. & Consciencia & - & & & & & & & & \\
\hline 2. & Transcendencia & .361 & - & & & & & & & \\
\hline 3. & Sentido & .374 & .469 & - & & & & & & \\
\hline 4. & Compasión-Amor & .358 & .592 & .427 & - & & & & & \\
\hline 5. & Perdón & -.181 & -.072 & -.237 & .027 & - & & & & \\
\hline 6. & Gratitud & .411 & .495 & .519 & .560 & -.155 & - & & & \\
\hline 7. & Dolor & .433 & .491 & .579 & .481 & -.299 & .515 & - & & \\
\hline 8. & Libertad & .467 & .432 & .619 & .410 & -.220 & .560 & .688 & - & \\
\hline 9. & Efectos & .374 & .346 & .553 & .410 & -.216 & .560 & .537 & .563 & - \\
\hline
\end{tabular}

Para valorar mejor el conjunto de relaciones entre los distintos factores se llevó a cabo un Análisis de Conglomerados. La Tabla 5 presenta la Matriz de distancias obtenida entre las competencias de la IES.

La medición de similitud permitió la obtención de los conglomerados y el dendograma muestra las vinculaciones medias entre los factores recogidas en la Figura 1.

Estos resultados muestran que los dos factores con mayor similitud en sus patrones de respuesta son los factores de Dolor y Libertad. Estas dos competencias muestran ma- yor similitud con Sentido que con el resto de factores, conformando un nuevo grupo de mayor similitud. Los resultados muestran también la mayor similitud entre los factores Transcendencia y Compasión-amor, así como entre Gratitud y Efectos. Por último, se agrupan Consciencia y Perdón.

Para verificar la validez de criterio se halló el coeficiente de correlación de los rasgos de Spearman y se relacionaron las puntuaciones de cada sujeto con las variables Autoestima y Resiliencia.

\section{Figura 1.}

Dendograma, vinculaciones medias entre los factores.

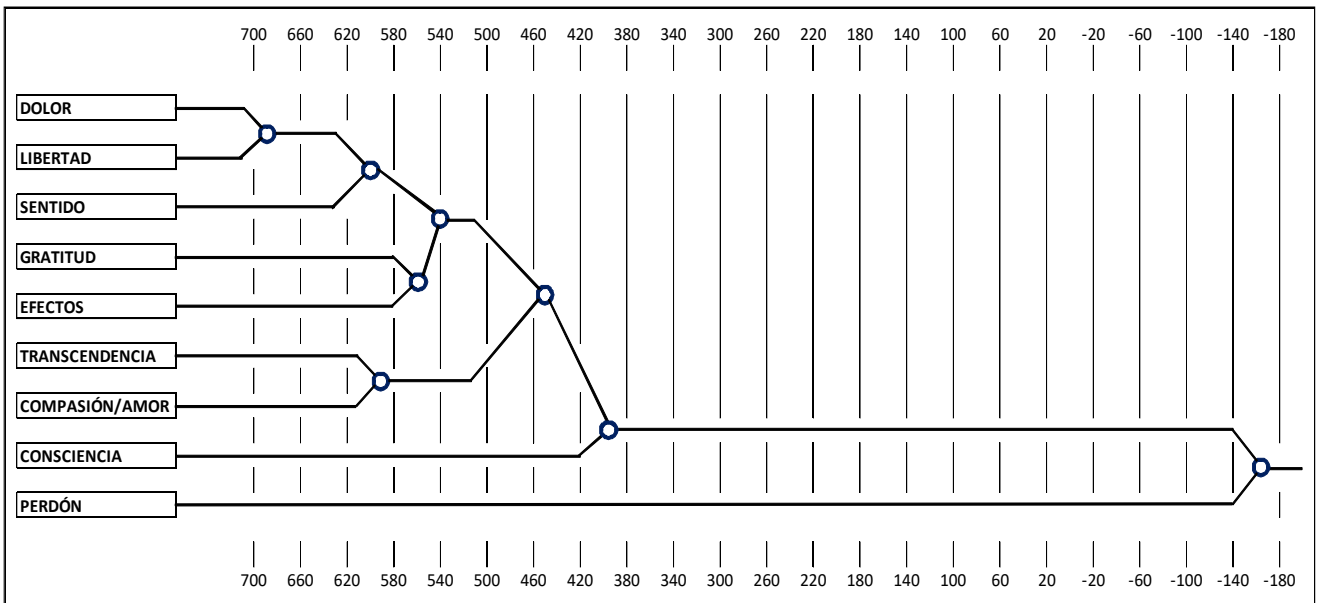


Estas variables criterio han sido seleccionadas en función del respaldo alcanzado por otros estudios de IES en la revisión bibliográfica realizada (Furnham y Ward, 2001; Keshavarzi y Yousefi, 2012; Narayanan y Jose, 2011; Olson, 2008) y de las garantías psicométricas que ofrecen los cuestionarios RSQ y CD-RISC- 10 .

Los resultados recogidos en la Tabla 6 , confirmaron la relación significativa entre la puntuación global del CIES y la Escala de Resiliencia. Los resultados señalan una correlación positiva entre ambos constructos observada por otros autores (Jung et al., 2016; Keshavarzi y Yousefi, 2012; Narayanan y Jose, 2011).

Tabla 6.

Análisis de correlaciones entre el CIES, Resiliencia y Autoestima (Rho de Spearman) ( $N=528)$

\begin{tabular}{llcc}
\hline & CIES & Resiliencia & Autoestima \\
\hline Resiliencia &. $\mathbf{3 6 4}^{* *}$ & - & \\
Autoestima & -.011 & .085 & - \\
\hline
\end{tabular}

En cambio, no aparece una relación significativa entre la puntuación global del CIES y el Cuestionario de Autoestima. Sin embargo, cuando se relacionaron las escalas de Autoestima con cada uno de los factores del CIES correlaciona positivamente con Sentido y con Efectos y ne- gativamente con Perdón. Este hallazgo es consistente con distintas investigaciones (Benedict-Montgomery, 2014; Furnham y Ward, 2001; Neto y Furnham, 2011; Olson, 2008). Olson (2008), concretamente, establece una correlación positiva entre bienestar espiritual (Efectos) y autoestima.

La correlación con los datos de las Escalas de Resiliencia y Autoestima aportan nueva evidencia empírica sobre la validez del CIES.

En el análisis de regresión multivariante realizado se obtuvo un coeficiente de determinación del $99.6 \%$. Los resultados de este análisis se presentan en la Figura 2.

La regresión es óptima, precisa y la proporción de varianza explicada es potente. Esto confirma los resultados obtenidos en el análisis factorial y muestra la capacidad de predicción de la puntuación global a partir de cada uno de los factores. En definitiva, es posible ofrecer una versión corta del CIES que explica el $80.6 \%$ de la información y consta de 37 ítems de las competencias Trascendencia, Perdón, Compasión-amor y Libertad. Aunque dada la extensión del cuestionario, se considera que es mejor utilizar la versión del CIES de 68 ítems que permite aportar un perfil competencial más completo.

\section{Figura 2.}

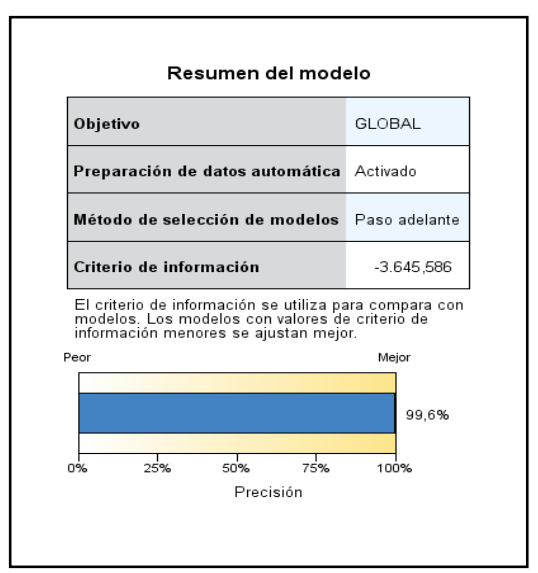


Además, se calculó el peso de cada uno de los factores independientemente de lo que comparten con los demás, mediante la aplicación de ecuaciones estructurales. De esta manera, se computó la contribución de cada factor en la puntuación global del CIES. Los resultados de este análisis se presentan en la Figura 3. Las diferencias entre los factores son escasas. El factor Trascendencia es el que más contribuye y el factor que menos Dolor aunque la mayoría están en torno al 0.10. Por tanto, la mayor diferencia se encuentra entre Dolor con 0.7 y Trascendencia con 1.7. En definitiva, todos los factores contribuyen a la puntuación global permitiendo concluir que el CIES es un cuestionario equilibrado.

\section{Figura 3.}

Distribución de las aportaciones de las competencias a la puntuación total del CIES

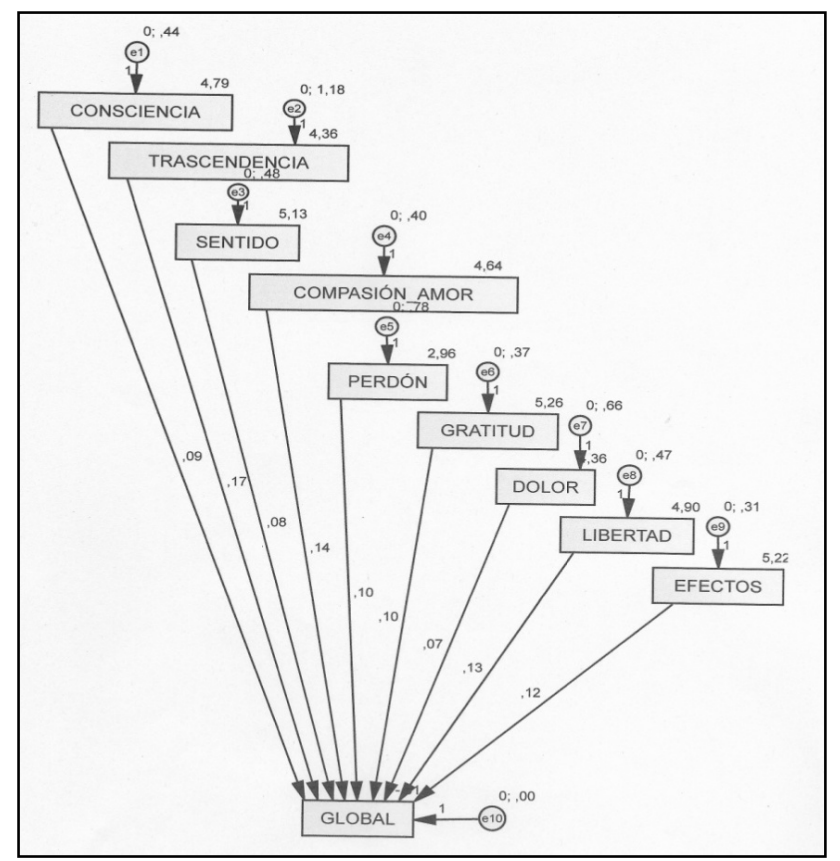

Adicionalmente, se llevó a cabo un proceso de baremación que para comparar la puntuación directa de la persona que realice el CIES con el grupo normativo que ha permitido la validación del mismo. La Tabla 7 presenta los resultados básicos de la baremación del CIES.
Tabla 7.

Resultados generales estadísticos de la baremación del CIES

\begin{tabular}{llr}
\hline Estadísticos & \\
\hline$N$ & Válido & 528 \\
Media & & 313.19 \\
Desviación estándar & & 32.93 \\
Mínimo & & 215 \\
Máximo & & 387 \\
Percentiles & 25 & 292 \\
& 50 & 315 \\
& 75 & 339 \\
\hline
\end{tabular}

El análisis de la media, la desviación típica, el mínimo y el máximo junto con los percentiles de la muestra determinaron el baremo del Cuestionario. Se establecieron tres niveles de competencia global en el cuestionario CIES: un nivel Inteligencia Espiritual bajo, (272 puntos o menos en la escala, medio (entre 273 y 340 puntos) y alto (341 o más puntos).

A partir del análisis diferencial con las nuevas categorías obtenidas en el proceso de baremación exploratoria del cuestionario, se deducen provisionalmente estas conclusiones:

- Los participantes que ingresan menos de 1000 euros mensuales puntúan bajo en el CIES, en cambio, los que tienen más de 3000 euros obtienen las puntuaciones más altas en la escala.

- Los participantes que viven en comunidad presentan puntuaciones más altas en el CIES.

- La IES es más baja hasta los 43 años, es media entre los 47-48 años y es alta a partir de los 50 años. Stockstill (2000), en su estudio sobre inteligencias múltiples, obtiene resultados similares: la madurez espiritual aumenta con la edad.

Este análisis diferencial ha permitido compararlos con otras investigaciones y variables sociodemográficas (sexo, edad, nivel económico, nivel formativo, estructura familiar) que confirman los resultados y añaden una nueva evidencia empírica de la validez del CIES. 


\section{Conclusiones}

Al inicio de esta investigación se planteó elaborar y validar un cuestionario que permitiera operativizar y evaluar la IES. Se puede concluir que se ha logrado este propósito; se ha definido el concepto de Inteligencia Espiritual, determinado nueve competencias y construido un instrumento de medida validado en la muestra obtenida.

El Análisis Factorial Exploratorio (AFE) ha permitido comprobar la validez de constructo. En el procedimiento de factorización aparecieron 14 factores con una varianza total explicada de más del $58 \%$. La dispersión de los datos permitió la agrupación de los ítems en 9 competencias. Estos están bien representados en el cuestionario.

En el análisis de regresión multivariante realizado se obtuvo que la regresión es óptima, precisa y la proporción de varianza explicada es potente. Esto confirma los resultados obtenidos en el análisis factorial y muestra la capacidad de predicción de la puntuación global a partir de cada uno de los factores.

Asimismo, el CIES es una escala equilibrada, en la que todos los factores contribuyen equilibradamente a la puntuación global.

El cuestionario CIES permite comparar la puntuación directa de la persona que lo realice con el grupo normativo de validación. El baremo del Cuestionario, al menos de manera exploratoria y provisional, permite diferenciar entre 3 niveles de Inteligencia Espiritual.

Finalmente, se examinaron las relaciones entre la IES, la resiliencia y la autoestima. La correlación con los datos de las Escalas de Resiliencia y Autoestima aportan evidencia empírica sobre la validez de criterio del CIES. Este hallazgo es consistente con distintas investigaciones similares (Benedict-Montgomery, 2014; Furnham y Ward, 2001; Neto y Furnham, 2011; Olson, 2008; Prieto-Ursúa el al., 2012). Aunque los resultados del análisis de la relación de la IES con la resiliencia y la autoestima, aportan evidencia empírica sobre la validez del cuestionario, indican también la conveniencia de seguir profundizando en el estudio de estas relaciones.
Sería interesante replicar los resultados de este estudio en muestras más amplias con porcentajes más altos de participantes de otras religiones y con un número más igualado de mujeres y hombres, que permitan generalizar aún más los resultados de la investigación.

De cara al futuro de este campo de investigación, el reto más arduo es la inclusión de la inteligencia espiritual y sus competencias en educación. Países anglosajones como Gran Bretaña y Canadá han incorporado el desarrollo de esta inteligencia en sus sistemas educativos (González, 2009). Reconocen así la importancia de estas competencias básicas para el desarrollo de todas las inteligencias y el crecimiento pleno e integral de toda persona. Un campo especialmente interesante de estudio es el diseño de los programas más efectivos para el cultivo y el desarrollo de cada una de las competencias: trabajar estas competencias espirituales hace posible una educación realmente más integral e integradora (Sisk, 2008; Sisk y Torrance, 2002). Sisk (2008) elaboró un programa de crecimiento en IES en secundaria, lo puso en práctica y midió los resultados. Los estudiantes obtuvieron puntuaciones más altas en el post-test que en el pre-test determinando así que el programa aumentó su conciencia y comprensión de los rasgos de la IES.

Distintas investigaciones (Hofmann y Walach, 2011; Magaldi-Dopman et al., 2011) informan de la importancia de la espiritualidad en los dos tercios de los psicoterapeutas con experiencia práctica, buena formación y acreditados, que trabajan tanto en el sistema público como privado, con resultados similares en Alemania y en Estados Unidos. Aunque en Europa y Alemania, en particular, se considera que los temas espirituales y religiosos tienen influencia sobre los individuos y consideran que es un gran recurso para la estabilidad y salud psicológica de las personas (Kohl et al., 2009; Kohls y Walach, 2007). Como se señala en estas investigaciones, un número significativo de psicoterapeutas consideran espiritualidad y religión fundamentales para el tratamiento, la práctica y la formación terapéutica. Los resultados de estas investigaciones confirman la necesidad de programas de formación de psicología y psicoterapia que presten atención adicional a la integración de dimensiones espirituales y religiosas. Los sesgos y prejuicios espirituales y religiosos de clientes y psicoterapeutas no han sido explorados ampliamente, pero 
algunas investigaciones sugieren su impacto en el trabajo terapéutico (Pesut et al., 2008). La espiritualidad y la religión están siendo incluidas en el ámbito de la terapia familiar. (Carlson et al., 2002; Erickson et al., 2002). Se aprecia una diferencia claramente significativa entre terapeutas de distintas orientaciones teóricas sobre la importancia que asignan a lo espiritual o religioso en la terapia $\mathrm{y}$ en la formación de los terapeutas y se constata que la supervisión en estos temas es un imperativo ético para el campo.

Igualmente, se considera importante para futuras investigaciones valorar la implicación de la IES en organizaciones y empresas. Ya hay iniciativas significativas en el liderazgo empresarial, la responsabilidad social corporativa y los modelos económicos sostenibles (Villafranca, 2016; Wigglesworth, 2013a, 2013b).

\section{Referencias}

Alonso, A. (2011). Pedagogía de la Interioridad [Pedagogy of Interiority]. Narcea.

Amram, Y. (2007, agosto). The Seven Dimensions of Spiritual Intelligence: An Ecumenical Grounded Theory. Paper presented at the 115th Annual Conference of the American Psychological Association, San Francisco, CA. http://www.yosiamram.net/papers

Amran, Y. y Dryer, D. C. (2008). The Integrated Spiritual Intelligence Scale (ISIS): Development and Preliminary Validation. Paper presented at the 116th Annual Conference of the American Psychological Association, Boston.

Benedict-Montgomery, M. M. (2014). Our Spirits, Ourselves: The relationships between Spiritual Intelligence, Self-compassion, and Life Satisfaction [Tesis doctoral], Alliant International University, USA.

Cabero, J. y Llorente, M. C. (2013). La aplicación del juicio de experto como técnica de evaluación de las tecnologías de la información (TIC) [The Experts Judgment Application as a Technic Evaluate Information and Communication Technology (ICT)]. Revista de Tecnología de Información y Comunicación en Educación, 7(2), 11-22.

Cloninger, C. R. (2004). Feeling Good: The Science of Well-Being. Oxford University Press.

Carlson, T. D., Kirkpatrick, D., Hecker, L. y Killmer, M. (2002). Religion, Spirituality, and Marriage and Family Therapy: A Study of Family Therapists' Beliefs about the Appropriateness of Addressing Religious and Spiritual Issues in Therapy. American Journal of Family Therapy, 30(2), 157171. https://doi.org/10.1080/019261802753573867

Chaktoura, E. (2015). Inteligencia espiritual: para atrevernos a vivir la vida que queremos. Grijalbo.

Connor, K. M. y Davidson, R. (2003). Development of a New Resilience Scale: The Connor-Davidson Resilience Scale (CD-RISC). Depress Anxiety, 18(2), 76-82. https://doi.org/10.1002/da.10113

Day, C. (2006). Pasión por enseñar. La identidad personal y profesional del docente y sus valores. Narcea.

Draper, B. (2010). La Inteligencia Espiritual: un nuevo modo de ser. Sal Terrae.

Emmons, R. A. (2000). Spirituality and Intelligence: Problems and Prospects. The International Journal for the Psychology of Religion, 10(1), 57-64. https://doi.org/10.1207/S15327582IJPR1001 6

Erickson, M. J., Hecker, L., Kirkpatrick, D., Killmer, M. y James, E. (2002). Clients' Perceptions of Marriage and Family Therapists Addressing the Religious and Spiritual Aspects of Clients' Lives: A Pilot Study. Journal of Family Psychotherapy, 13(1-2), 109-125. https://doi.org/10.1300/J085v13n01_06

Fernández-Montalvo, J. y Echeburúa, E. (2008). Trastornos de personalidad y psicopatía en hombres 
condenados por violencia grave contra la pareja [Personality Disorders and Psychopathy in Men Convicted for Severe Intimate Partner Violence]. Psicothema, 20(2), 193-198.

Furnham, A. y Ward, C. (2001). Sex Differences, Test Experience and the Self-estimation of Multiple Intelligences. New Zealand Journal of Psychology, $30(2), 52-59$.

Gómez, I. (2014). Educar la inteligencia espiritual. Recursos para la clase de Religión. Khaf.

González, P. (2009). Reflexiones en torno a la competencia espiritual: la dimensión espiritual y religiosa en el contexto de las Competencias Básicas Educativas. Escuelas Católicas.

Hair, J. F., Anderson, R. E., Tatham, R. L. y Black, W. C. (2007). Análisis multivariante [Multivariate Analysis]. Pearson.

Hofmann, L., y Walach, H. (2011). Spirituality and Religiosity in Psychotherapy. A Representative survey among German psychotherapists. Psychotherapy Research, 21(2), 179-192. https://doi.org/10.1080/10503307.2010.536595

Jahani (2010). The Relationship between Self efficacy and Irrational Beliefs with General Health among University Students of Imam Khomeini University. International University of Imam Khomeini.

Jung Y. H., Ha T. M., Oh C. Y., Lee U. S., Jang J. H., Kim J., Park J. O. y Kang D. H. (2016). The Effects of an Online Mind-Body Training Program on Stress, Coping Strategies, Emotional Intelligence, Resilience and Psychological State. PLoS ONE, 11(8), $1-20$. https://doi.org/10.1371/journal.pone.0159841

Keshavarzi, S. y Yousefi, F. (2012). The Relationship between Emotional Intelligence, Spiritual Intelligence, and Resilience. Journal of Psychology, 16(3), 299-318.
King, D. B. (2008). Rethinking Claims of Spiritual Intelligence: A definition, Model, and Measure [Tesis de Máster], Trent University, Canada.

King, D. B. y DeCicco, T. L. (2009). A Viable Model and Self-report Measure of Spiritual Intelligence. International Journal of Transpersonal Studies, 28(1), 68-85.

Kohls, N. y Walach, H. (2007). Psychological Distress, Experiences of Ego Loss and Spirituality: Exploring the Effects of Spiritual Practice. Social Behavior and Personality, 35(10), 1301-1316 https://doi.org/10.2224/sbp.2007.35.10.1301

Kohls, N. Walach, H. y Wirtz, M. (2009). The Relationship between Spiritual Experiences, Transpersonal Trust, Social Support, and Sense of Coherence and Mental Distress - A Comparison of Spiritually Practising and Non-Practising Samples. Mental Health, Religion \& Culture, 12(1), 1-23.

Lima Rodríguez, J. S., Lima Serrano, M., Jiménez Picón, N. y Domínguez Sánchez, I. (2012). Consistencia interna y validez de un cuestionario para medir la autopercepción del estado de salud familiar [Reliability and Construct Validity of an Instrument to Asses the Self-perception of Family Health Status]. Revista Española de Salud Pública, 86(5), 509-521.

MacGilchrist, B., Reed, J. y Myers, K. (2004). The Intelligent School. Sage.

Magaldi-Dopman, D., Park-Taylor, J. y Ponterotto, J. G. (2011). Psychotherapists' Spiritual, Religious, Atheist or Agnostic Identity and their Practice of Psychotherapy: A Grounded Theory Study. Psychotherapy Research, 21(3), 286-303. https://doi.org/10.1080/10503307.2011.565488.

Martín-Sánchez, A., Rodríguez-Zafra, M. y CenicerosEstévez, J. C. (2020). Definición y Competencias de la Inteligencia Espiritual. Estudio Cualitativo [Definition and Competences of Spiritual Intelligence. Qualitative Study]. Acción 
Psicológica,

$17(2)$

https://doi.org/10.5944/ap.17.2.29527

Martínez-Arias, R. Castellanos, M.A. y Chacón, J.C. (2014). Métodos de Investigación en Psicología [Research Methods in Psychology]. EOS.

Maxim, M. (2008). Spiritual Intelligence in Executive Coaching. Journal of Evidence Based Coaching and Mentoring. Special Issue 4, October.

Narayanan, A. y Jose, T. P. (2011). Spiritual intelligence and resilience among Christian youth in Kerala. Journal of the Indian Academy of Applied Psychology, 37(2), 263-268.

Neto, F. y Furnham, A. (2011) Sex Differences in Parents' Estimations of their Own and their Children's Multiple Intelligences: A Portuguese Replication. The Spanish Journal of Psychology, 14 (1), 99-110. https://doi.org/10.5209/rev_SJOP.2011.v14.n1.8

Noble, K. D. (2000). Spiritual Intelligence: A New Frame of Mind. Advanced Development Journal, 9, $1-29$.

Olson, L. G. (2008). An Investigation of Factors that Influence Academic Achievement in Christian Higher Education: Emotional Intelligence, Selfesteem, and Spiritual Well-being [Tesis doctoral], Capella University, USA.

Pesut, B., Fowler M., Taylor E. J, Reimer-Kirkham, S. y Sawatzky R. (2008). Conceptualising Spirituality and Religion for Healthcare. Journal of Clinical Nursing, $\quad$ 17(21), 2803-2810. https://doi.org/10.1111/j.1365-2702.2008.02344.x

Peterson, C., y Seligman, M. E. P. (2004). Character Strengths and Virtues: A Classification and Handbook. Oxford University Press.

Prieto-Ursúa, M., Galán, M. J. C., de Gregorio, V. C., Gismero, E., González, M., y San Roque, I. M. (2012). El Perdón como Herramienta Clínica en
Terapia Individual y de Pareja [Clinical Use of Forgiveness in Individual and Marital Therapy]. Clínica, 3(2), 121-134.

Ronel, N. (2008). The Experience of Spiritual Intelligence. Journal of Transpersonal Psychology, 40(1), 100-119.

Rosenberg, M. (1965). Society and the Adolescent SelfImage. Princeton University Press.

Serrano-Parra, M. D., Garrido-Abejar, M., NotarioPacheco, B., Bartolomé-Gutiérrez, R., SoleraMartínez, M., y Martínez-Vizcaíno, V. (2013). Validez de la Escala de Resiliencia de ConnorDavidson (10 ítems) en una población de mayores no institucionalizados [Validity of the ConnorDavidson Resilience Scale (10 items) in a Population of Elderly]. Enfermería Clínica, 23(1), 14-21. https://doi.org/10.1016/j.enfcli.2012.11.006

Sisk, D. A. y Torrance, E. P. (2002). Spiritual Intelligence: Developing Higher Consciousnesses. Creative Education Foundation Press.

Sisk, D. (2008). Engaging the Spiritual Intelligence of Gifted Students to Build Global Awareness in the Classroom. Roeper Review A Journal on Gifted Education, $\quad 30(1), \quad 24-30$. https://doi.org/10.10800278319070

Stockstill, D. B. (2000). Intelligence and Faith in Adolescents: A Study of the Relationship between Multiple Intelligences and Faith Formation during Adolescence [Tesis doctoral], The Union Institute, USA.

Torralba, F. (2010). Inteligencia Espiritual [Spiritual Intelligence]. Plataforma.

Vázquez, J. L. (2012). Contemplación e Inteligencia Espiritual [Contemplation and Spiritual Intelligence]. Imprenta Kadmos. 
Vaughan, F. (2002). What is Spiritual Intelligence? Journal of Humanistic Psychology, 42(2), 16-33.

Villafranca, E. (2016). La Responsabilidad Social, Producto de Nuestra Inteligencia Espiritual: Lecciones aprendidas para crear negocios sostenibles. Jonathan Sibaja Rodríguez.

Wigglesworth, C. (2013a). Las 21 aptitudes de la inteligencia espiritual: un paso más allá de la inteligencia emocional. Grijalbo.

Wigglesworth, C. (2013b). Spiritual Intelligence. En J. Neal (Ed.), Handbook of Faith and Spirituality in the Workplace: Emerging Research and Practice (pp. 441-453). Springer.

Wolman, R. N. (2001). Inteligência espiritual [Spiritual Intelligence]. Ediouro.

Zohar, D. y Marshall, I. (2000). Inteligencia Espiritual [Spiritual Intelligence]. Plaza \& Janes.

\section{Anexo A}

\section{GUESTIONARIO DE INTELIGENGIA ESPIRITUAL (GIES). VERSIÓN DEFINITIVA. \\ Competencias del CIES \\ ITEMS}

\section{Consciencia}

1. Puedo concentrarme y escuchar con atención y profundidad.

2. Me doy cuenta de lo que experimento interiormente durante una conversación o una tarea.

3. Puedo aceptar aspectos aparentemente contradictorios de la vida.

4. Siento mi propia vulnerabilidad.

5. Puedo diferenciar mis sentimientos de los sentimientos de otras personas.

6. Acepto mis limitaciones: ni puedo, ni sé todo.

\section{Trascendencia}

7. Soy capaz de descubrir la presencia del Misterio en las personas, en la vida, en el universo.

8. Recurro a la confianza, a la fe en una fuerza que va más allá de mí cuando enfrento los problemas de la vida.

9. Me apoyo en algunas experiencias extraordinarias o trascendentes para lidiar con los problemas cotidianos.

10. Tengo experiencias sensitivas o espirituales que muestran una unidad profunda a pesar de la aparente separación.

11. Experimento una conexión con Alguien o con Algo mayor.

12. Comprometerme con alguien o con algo que va más allá de mí, me ayuda a ir descubriendo aspectos de mí mismo.

13. Creo que existe una dimensión trascendente, espiritual en las personas, en la vida.

14. Siento una Presencia amorosa que envuelve todo.

15. Practico algún método que me ayuda a ser más consciente (meditación, contemplación, atención plena, relajación...).

16. Creo que la capacidad de amar de verdad es la clave de toda espiritualidad auténtica.

17. Busco la serenidad interior y exterior como un modo de estar en la vida.

18. Me hago preguntas sobre la existencia, el dolor, la muerte, el bien o el mal y busco respuestas. 


\section{Sentido}

19. Aprendo de las dificultades pasadas y las utilizo para afrontar nuevas dificultades.

20. Me merece la pena seguir viviendo: encuentro motivos cada día.

21. Tomo decisiones que van conformando mi vida hacia un propósito.

22. Encontrar un sentido en la vida me ayuda a afrontar las situaciones dolorosas.

23. Afronto la vida con confianza y esperanza.

\section{Compasión-Amor}

24. Creo que el amor a los demás está relacionado con el amor a sí mismo.

25. El amor busca el bien común con actuaciones concretas.

26. Necesito una persona que me guíe y me proteja.

27. Siento compasión por los sufrimientos y las esperanzas que todos compartimos, aunque tengamos una visión de la vida diferente.

28. Me comprometo responsablemente de acuerdo con mis creencias y mis principios.

29. Me duele el sufrimiento de otras personas y deseo poder aliviarlo.

30. Dedico tiempo y recursos a ayudar a otras personas sin esperar que hagan lo mismo conmigo.

31. Pido ayuda para aliviar o acabar con el dolor.

32. Me siento mejor cuando comparto mis penas y encuentro apoyo y consuelo.

\section{Perdón}

33. Soy incapaz de perdonarme mis fallos y errores.

34. Me critico continuamente cuando hago algo mal.

35. Siento una rabia, un resentimiento continuo hacia quienes me han hecho daño.

36. Cuando me hacen daño pienso en ello una y otra vez.

37. Guardo todas las situaciones negativas que he sufrido sin pasar página.

38. Dedico mucho tiempo a ver si los demás me quieren o me valoran.

39. Cuando estoy lejos de las personas que amo siento inquietud, angustia y necesito estar en contacto con ellas constantemente.

\section{Gratitud}

40. Agradezco mucho cuando personas que apenas conozco me ayudan amablemente.

41. Valoro que alguien haga algo por mí, aunque sea sólo un gesto.

42. Doy las gracias a menudo cuando me ayudan en algo.

43. Me doy cuenta de las muchas cosas que he de agradecer.

44. Soy consciente de las pequeñas cosas que me ocurren y que son para mí un verdadero regalo.

45. Incluso en momentos dolorosos de mi vida, siento agradecimiento por haber tenido fuerzas para superarlo o haber encontrado un sentido.

46. Siento un impulso fuerte para seguir creciendo más plenamente.

\section{Dolor}

47. Me doy ánimo para aguantar las experiencias dolorosas que no puedo evitar.

48. Tengo recursos personales y sociales para enfrentar las experiencias dolorosas que acontecen en la vida.

49. Puedo llegar a comprender a personas que me han herido.

50. Puedo tomar cierta distancia de las circunstancias y elegir cómo reaccionar. 
51. Siento paz aún en medio de situaciones dolorosas o estresantes.

\title{
Libertad
}

52. Dedico tiempo para darme cuenta de lo que me condiciona y lo que quiero realmente cuando tomo decisiones importantes.

53. Tengo mi propio código de valores que orienta mi conducta.

54. Me siento más libre cuanto más me conozco y más comprendo mi propia historia y mis circunstancias.

55. Puedo elegir, al menos, la actitud con la que vivir las circunstancias que me tocan.

56. Siento que soy el dueño de mi vida a pesar de condicionamientos, factores externos o internos.

57. Me he liberado de falsas creencias que aprendí en mis otras etapas de mi vida.

58. Busco conocerme y comprenderme cada vez más y mejor.

59. Al tomar una decisión tengo en cuenta lo que intuyo, lo que pienso y lo que siento

60. Intento saber qué puedo hacer para evitar el sufrimiento inútil.

\section{Efectos de la IES}

61. Confío que, al final, todo irá bien.

62. Me responsabilizo de mis ideas, sentimientos y comportamientos.

63. Soy feliz cuando hago algo bueno por los demás.

64. El sentido del humor me permite relativizar los problemas.

65. Aprecio la belleza que encuentro en las personas, la vida...

66. Disfruto las pequeñas cosas de la vida como un paseo o una comida.

67. Tengo relaciones significativas y duraderas que cuido.

68. Sé que las experiencias dolorosas forman parte de la vida tarde o temprano.

\section{THE STUDY OF SPIRITUAL INTELLIGENCE II: AN INSTRUMENT FOR EVALUATING THEIR COMPETENCE PERFORMANGE}

\author{
ANTONIA MARTÍN-SÁNCHEZ, MóNICA RODRÍGUEZ-ZAFRA Y \\ JuAN CARLOS CENICEROS-ESTÉVEZ ${ }^{1}$
}

\section{EXTENDED SUMMARY}

In the first part of this research, Martín-Sánchez et al. (2020), literature on Spiritual Intelligence (SI) was analyzed and, jointly with the qualitative study, has allowed to define and specify the SI competences. After this research, Spiritual Intelligence was defined as a system of capacities necessary for the deep understanding of questions of meaning and meaning that make possible the in- tegration of all the dimensions of the person, the resolution of existential problems, the transcendence and the transformation of the everyday reality through high levels of consciousness and ethical coherence. This intelligence was operationalized in nine competencies: Consciousness, Transcendence, Meaning, Compassion-love, Acceptance of pain, Forgiveness, Gratitude, Freedom and Effects.

In this second study, the process and results of the construction of a questionnaire for the measurement of SI are presented and the results of the verification and contrast phase of the results are collected. 
Thanks to the analysis of the study of the concept of SI carried out from the existing literature and to the qualitative research collected in Martín-Sánchez et al. (2020), the first version of the questionnaire was prepared. 118 items were drafted that operationalize the nine competencies that make up the definition of SI. The first version of the questionnaire was subjected to the following analyzes and checks of theoretical and technical quality to ensure the validity and reliability of the data, before its final application: Validity of the design and content of the questionnaire, Analysis of the reliability and internal consistency of the questionnaire (Pilot test) and Validation of the construct.

The content validation process was carried out by submitting the questionnaire to the Expert Judgment Technique and, specifically, to the individual aggregate method (Cabero \& Llorente, 2013). To do this, each expert was asked individually to give a direct estimate of the probability of success or failure in the proposed demographic data and in each of the items describing the competencies of the SI. To this end, the CIES evaluation instrument was developed for the validation of the experts. In it, they were presented with the objective of the research together with the recipients of the same, the sections of the instrument (demographic data and the items associated with each of the estimated competences of the SI) and the additional norms for validation. Next, they were asked to rate the defining items for each competency (the relevance and clarity of each of them on a scale of 1 to 5) and make observations. This document was sent to six specialist experts in Spirituality, Psychology and Research Methods. All the items valued below four points were discarded and all the contributions made by the experts in the writing of any of the statements were collected. Therefore, CIES was reduced to 104 items out of 118 initials.

After validation of the design and the contents of the questionnaire by the six experts specialized in Spirituality, Psychology and Research Methods, its reliability and internal consistency were assessed through a pilot test. The sample for this pilot test consisted of 53 participants selected by the snowball procedure. To do this, an online questionnaire was constructed and sent to an initial group of participants who were asked to forward it, in turn, to other people. Once the sociodemographic data of the pilot sample had been reviewed, the total reliability of the questionnaire and the reliability of each of the competences were calculated. The global Cronbach's alpha, 0.888, was estimated, a high value that indicates a high reliability of the questionnaire that allows us to deduce that all the items measure the same aspect. To perform the analysis and selection of the most representative items for each competence, the correlation of each item with the total was found. The criteria used to select the items was to eliminate those that least correlated with the total and their absence improved the internal consistency achieved. The global Cronbach's alpha was, in this pilot test, 0.888 with the 104 items and 0.941 with the 68 items finally selected. The reliability index of the competences of this latest version of the CIES is situated in a range that goes from 0.652 to 0.921 .

Once the questionnaire had been refined with the data obtained in the pilot test, the last phase of validation of the questionnaire was carried out. The definitive version of the CIES online was built and sent to a series of participants and they were asked to forward it, in turn, to other people, following the non-probability sampling procedure, as in the pilot test. A snowball sampling was chosen and, therefore, the fundamental criterion in the selection of the sample was accessibility. In the initial submissions, heterogeneous informants were sought, representative of various spiritual currents, ages, sexes, economic and educational levels. The anonymity of the participants and the confidentiality of the demographic data requested were guaranteed, as well as respect for the ethical principles that all research must respect (Martínez-Arias et al., 2014).

The statistical package SPSS Statistics 24 was used for the analysis of the data collected in the database. For the differential analysis a confidence level of $95 \%$ was taken into account.

The final sample consisted of 528 participants, 132 men and 396 women, which represents $25 \%$ and $75 \%$ respectively in percentages. The variability of the ages of the participants ranged between 18 and 88 years, the average being 47 years of age, with a standard deviation of 13 . Regarding the economic level, $76 \%$ did not exceed a salary of 2000 euros per month and less than $6 \%$ exceeded 3000 
euros per month. The level of study of the participants was high: $81 \%$ had higher and / or postgraduate training; only $3 \%$ had primary education. Regarding the religious trend or religion, the largest group defined themselves as Catholic Christian with $68 \%$, followed by those who affirm that they did not ascribe to any religion with $20 \% .2 \%$ acknowledged that they practiced all or that they kept "the best of each one". The other religions complete the rest. The average number of people with whom the participants lived was two; and, regarding the place of residence, $81 \%$ of the sample lived in a city compared to $19 \%$ who lived in a town.

A descriptive analysis of the SI scores and the rest of the variables and an inferential analysis were carried out to find possible differences between the variables. For the construct validity, the Exploratory Factor Analysis-EFA was applied and, to verify the criterion validity, the Spearman Rho correlation coefficient was calculated and the correlations in the scores of each subject were confirmed with the measures of Self-esteem and Resilience.

Regarding the analysis of the reliability and internal consistency of the CIES, the data support the internal consistency of the questionnaire. The global Cronbach's alpha was 0.925 . The internal consistency of the ability subscales was also high: Consciousness 0.680; Transcendence 0.902; Sense 0.748; Love 0.678; Sorry 0.772; Gratitude 0.785; Acceptance of Pain 0.741; Freedom 0.781 and Effects 0.751 .

The Exploratory Factor Analysis (EFA) allowed to check the construct validity. Reliability (internal consistency) was determined with a Cronbach's alpha of 0.925. For the different competences, a Cronbach's alpha between 0.650 and 0.750 is acceptable and between 0.750 and 0.899 is very good. All the dimensions were in the latter interval or very close after the reorganization of the items. Likewise, the unidimensionality of the questionnaire was determined following the criteria of Lima et al. (2012).

In the factoring procedure, 14 factors appeared with a total explained variance of $58.12 \%$. The next step was to check the grouping of the 68 items in the 14 factors in the matrix obtained from the Varimax rotation. The dispersion of the data allowed the grouping of the items. These were well represented, with values higher than 0.5 in the vast majority of cases or close to this value. After the distribution and grouping of the factors by competences, the latest version of the questionnaire was obtained.

The most valued competence was Gratitude, followed by the Effects or consequences derived from a high spiritual intelligence. In addition to these two, Sense, Freedom, Consciousness and Compassion-love are above average; Transcendence, Acceptance of pain and Forgiveness below. The latter is the one that obtained the lowest score, with a significant difference of more than two points.

The CIES questionnaire makes it possible to compare the direct score of the person who performs it with the normative validation group. The scale of the Questionnaire, at least in an exploratory and provisional way, allows to differentiate between 3 levels of Spiritual Intelligence.

Facing the future of this field of research, the most arduous challenge is the inclusion of SI and its competences in education. Anglo-Saxon countries such as Great Britain and Canada have incorporated the development of this intelligence in their educational systems (González, 2009). They thus recognize the importance of these basic competencies for the development of all intelligences and the full and comprehensive growth of every person. A particularly interesting field of study is the design of the most effective programs for the cultivation and development of each of the competencies: working on these spiritual competencies makes a truly more comprehensive and inclusive education possible (Sisk, 2008; Sisk \& Torrance, 2002). Sisk (2008) developed a growth program in secondary schools, put it into practice and measured the results. The students obtained higher scores in the post-test than in the pre-test, thus determining that the program increased their awareness and understanding of the features of the SI.

Different investigations (Hofmann \& Walach, 2011; Magaldi-Dopman et al., 2011) report the importance of spirituality in two thirds of psychotherapists with practical experience, good training and accredited, who work in both the public and private systems, with similar results in Germany and the United States. Although in Europe and 
Germany, in particular, spiritual and religious issues are considered to influence individuals and are considered to be a great resource for the stability and psychological health of people (Kohl et al., 2009; Kohls \& Walach, 2007). As noted in these investigations, a significant number of psychotherapists consider spirituality and religion fundamental to treatment, practice and therapeutic training. The results of these investigations confirm the need for psychology and psychotherapy training programs that pay additional attention to the integration of spiritual and religious dimensions. The spiritual and religious biases and biases of clients and psychotherapists have not been widely explored, but some research suggests their impact on therapeutic work (Pesut et al., 2008). Spirituality and religion are being included in the realm of family therapy. (Carlson et al., 2002; Erickson et al., 2002). There is a clearly significant difference between therapists of different theoretical orientations on the importance they assign to the spiritual or religious in therapy and in the training of therapists and it is verified that supervision in these issues is an ethical imperative for the field.

Likewise, it is considered important for future research to assess the involvement of SI in organizations and companies. There are already significant initiatives in business leadership, corporate social responsibility, and sustainable economic models (Villafranca, 2016; Wigglesworth, 2013a, 2013b). 